\title{
Review of AdS/CFT Integrability, Chapter III.5: Lüscher Corrections
}

\author{
ROMUALD A. JANIK \\ Institute of Physics, Jagiellonian University, ul. Reymonta 4, 30-059 Kraków, Poland. \\ e-mail: romuald@th.if.uj.edu.pl
}

Received: 14 December 2010 / Revised: 26 March 2011 / Accepted: 5 April 2011

Published online: 19 July 2011 - (C) The Author(s) 2011. This article is published with open access at Springerlink.com

\begin{abstract}
In integrable quantum field theories, the large volume spectrum is given by the Bethe Ansatz. The leading corrections, due to virtual particles circulating around the cylinder, are encoded in so-called Lüscher corrections. In order to apply these techniques to the AdS/CFT correspondence, one has to generalize these corrections to the case of generic dispersion relations and to multiparticle states. We review these various generalizations and the applications of Lüscher's corrections to the study of the worldsheet QFT of the superstring in $A d S_{5} \times S^{5}$ and, consequently, to anomalous dimensions of operators in $\mathcal{N}=4$ SYM theory.
\end{abstract}

Mathematics Subject Classification (2010). 81T30, 81T40, 81U15, 81U20.

Keywords. AdS/CFT correspondence, integrability, integrable quantum field theory.

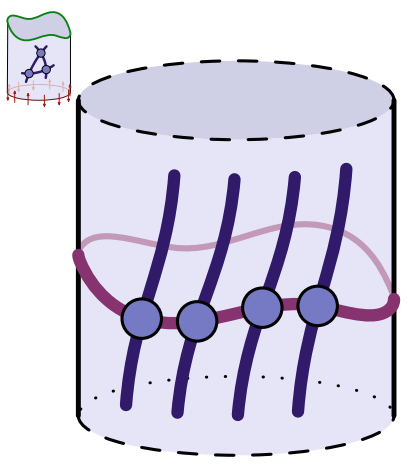

\section{Introduction}

For many integrable systems, the main question that one is interested in is the understanding of the energy spectrum for the system of a given size $L$. The size of the system in question may be discrete, like the number of sites of a spin chain 
or other kind of lattice system, or continuous, like the circumference of a cylinder on which a given integrable field theory is defined.

The first answer to this question for a wide variety of integrable systems is generically given in terms of Bethe equations. These are equations for a set of (complex) numbers $p_{i}$ of the form

$$
1=\mathrm{e}^{i p_{j} L} \prod_{k: k \neq j}^{N} S\left(p_{j}, p_{k}\right)
$$

Once a solution $\left\{p_{j}\right\}_{j=1, \ldots, N}$ is found, the energy is obtained through an additive formula

$$
E=\sum_{j=1}^{N} E\left(p_{j}\right)
$$

where $E(p)$ and $S\left(p, p^{\prime}\right)$ are (known) functions characterizing the given integrable system. In practice, for generic integrable systems, these equations become the more complicated nested Bethe equations, with a system of equations instead of (1.1), with additional auxiliary unknowns appearing in (1.1) but not in the energy formula (1.2). All this is described in detail in two other chapters of this review $[1,2]$.

Bethe equations of the type described above appear both in the case of discrete integrable spin chains and continuous two-dimensional integrable quantum field theories. Moreover, they also appear as equations for the anomalous dimensions of single trace operators in the $\mathcal{N}=4$ four-dimensional SYM theory and in various other contexts.

Now comes the fundamental difference between the various classes of integrable systems. For integrable spin chains, like the Heisenberg XXX, XXZ etc. models, the Bethe ansatz equations are exact and the energies given by (1.2) are the exact eigenvalues of the spin chain hamiltonian. On the other hand, for two-dimensional integrable quantum field theories, the answer provided by (1.1) and (1.2) is only valid for asymptotically large sizes of the cylinder $L$. There are corrections which arise due to the quantum field theoretical nature of the system, namely virtual particles circulating around the cylinder and their interaction with the physical particles forming a given energy state. For a single particle in a relativistic QFT, Lüscher derived formulas [3] for the leading corrections. The goal of this chapter is to review the subsequent generalizations and applications of Lüscher corrections within the AdS/CFT correspondence. Let us note in passing that there may be also some intermediate cases like the Hubbard model as considered in [4], where the situation is not so clear.

Once one goes beyond these leading corrections by say decreasing the size of the system, one has to include the effects of multiple virtual corrections which becomes quite complicated, and have never been attempted so far. Fortunately, for integrable quantum field theories, there exists a technique of Thermodynamic 
Bethe Ansatz - TBA [5] (and/or nonlinear integral equations - NLIE [6,7]), which allows for finding the exact energy spectrum and thus effectively resuming all these virtual corrections. This is, however, technically very involved, so even for the cases where it is known, Lüscher corrections remain an efficient calculational tool. These exact treatments are described in the chapters [8] and [9] of this review.

As a final note, let us mention that for anomalous dimensions in the $\mathcal{N}=4$ SYM theory, the Bethe equations break down due to so-called wrapping interactions. This will be discussed in more detail in Section 3 (see also the chapter [10]). Since according to the AdS/CFT correspondence anomalous dimensions are exactly equal to the energies of string states in $A d S_{5} \times S^{5}$, which are just the energy levels of the two-dimensional integrable worldsheet quantum field theory, this violation of Bethe ansatz equations is in fact quite natural and can be quantitatively described using the formalism of Lüscher corrections for two-dimensional QFT.

The plan of this chapter is as follows. First, after introducing Lüscher's original formulas, we will describe the various derivations of (generalized versions of) these formulas - a diagrammatic one, through a large volume expansion of TBA equations and through a Poisson resummation of quadratic fluctuations. Then we will review recent applications of generalized Lüscher corrections within the context of the AdS/CFT correspondence.

\section{Lüscher Formulas}

Lüscher derived universal formulas for the leading large $L$ mass shift (w.r.t. the particle mass in infinite volume) of a single particle state when the theory is put on a cylinder of size $L$. The universality means that the value of the leading correction is determined completely by the infinite volume $S$-matrix of the theory. This relation does not depend on integrability at all, and is even valid for arbitrary QFT's in higher number of dimensions, however it is most useful for two-dimensional integrable field theories for which we very often know the exact analytical expression for the $S$-matrix.

The leading mass correction is given as a sum of two terms - the $F$-term

$$
\Delta m_{F}(L)=-m \int_{-\infty}^{\infty} \frac{\mathrm{d} \theta}{2 \pi} \mathrm{e}^{-m L \cosh \theta} \cosh \theta \sum_{b}\left(S_{a b}^{a b}\left(\theta+i \frac{\pi}{2}\right)-1\right)
$$

and the $\mu$-term

$$
\Delta m_{\mu}(L)=-\frac{\sqrt{3}}{2} m \sum_{b, c} M_{a b c}(-i) \operatorname{res}_{\theta=2 \pi i / 3} S_{a b}^{a b}(\theta) \cdot \mathrm{e}^{-\frac{\sqrt{3}}{2} m L}
$$

quoted here, for simplicity, for a two-dimensional theory with particles of the same mass [11]. $S_{a b}^{a b}(\theta)$ is the (infinite volume) $S$-matrix element, and $M_{a b c}=1$ if $c$ is a bound state of $a$ and $b$ and zero otherwise. These two terms have a distinct spacetime interpretation depicted in Figure 1. The $F$-term corresponds to the 

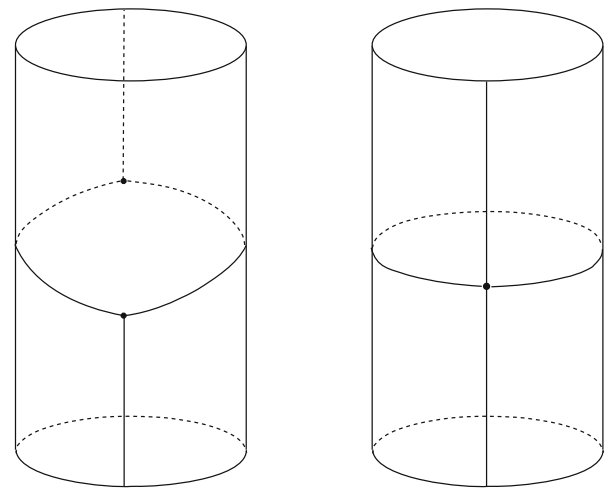

Figure 1. Spacetime interpretation of Lüscher's formulas - $\mu$-term (left) and $F$-term $($ right).

interaction of the physical particle with a virtual particle circulating around the cylinder, while the $\mu$-term corresponds to the splitting of the particle into two others which will then recombine after circulating around the cylinder.

In order to apply the above formulas to the case of the worldsheet QFT of the superstring in $A d S_{5} \times S^{5}$ (in generalized light cone gauge - see [12] for a detailed review), one has to generalize Lüscher's original formulas in two directions.

Firstly, the worldsheet QFT is not relativistic. The dispersion relation for elementary excitations is

$$
E(p)=\sqrt{1+16 g^{2} \sin ^{2} \frac{p}{2}}
$$

and moreover, there is no analog of a Lorentz symmetry, which brings about the fact that the $S$-matrix is a nontrivial function of two independent momenta instead of just the rapidity difference $\theta \equiv \theta_{1}-\theta_{2}$ as in the case of relativistic theories. Secondly, due to the level matching condition of the string, the physical states, corresponding to operators in $\mathcal{N}=4 \mathrm{SYM}$, have vanishing total momentum (or a multiple of $2 \pi$ ). Since single particle states with $p=0$ are protected by supersymmetry, all states interesting from the point of view of gauge theory are necessarily multiparticle states.

Consequently, one has to generalize Lüscher corrections to theories with quite generic dispersion relations and also to multiparticle states.

We will describe these generalizations at the same time showing how Lüscher corrections can be derived in many different and apparently unrelated ways.

\subsection{DIAGRAMMATIC DERIVATION}

The diagrammatic derivation was the original one used by Lüscher [3]. Its advantage is that it is the most general, does not assume integrability and is even valid in any number of dimensions. Its drawback, however, is that it is very difficult to 
generalize to multiparticle states or higher orders. On the other hand, it is easy to extend to theories with generic dispersion relations which was done in [13]. We will present a sketch of this derivation here applicable to a theory with the dispersion relation

$$
E^{2}=\varepsilon^{2}(p)
$$

which encompasses both the relativistic dispersion relation $\varepsilon^{2}(p)=m^{2}+p^{2}$, as well as the AdS one $\varepsilon^{2}(p)=1+16 g^{2} \sin ^{2} p / 2$.

The starting point is the observation that the dispersion relation is encoded, as the mass shell condition, in the pole structure of the Green's function. Hence to find the leading large $L$ corrections, one has to evaluate how the Green's function is modified at finite volume. It is convenient to translate the problem into a modification of the 1-particle irreducible (1PI) self energy defined by

$$
G(p)^{-1}=\varepsilon_{E}^{2}+\varepsilon^{2}(p)-\Sigma_{L}(p)
$$

The renormalization scheme is fixed by requiring that the self energy and its first derivatives vanish on-shell (at infinite volume). The shift of the energy, following from (2.5) becomes

$$
\delta \varepsilon_{L}=-\frac{1}{2 \varepsilon(p)} \Sigma_{L}(p)
$$

The propagator in a theory at fixed circumference can be obtained from the infinite volume one through averaging over translations $x \rightarrow x+n L$. In momentum space, this will correspond to distributing factors of $\mathrm{e}^{i n p^{1} L}$ over all lines. In the next step, we assume, following [3,11], that the dominant corrections at large $L$ will be those graphs which have only a single such factor with $n= \pm 1$. Picking $n=-1$ for definiteness, any such graph belongs to one of the three classes shown in Figure 2. Thus

$$
\Sigma_{L}=\frac{1}{2}\left(\sum_{b c} I_{a b c}+\sum_{b c} J_{a b c}+\sum_{b} K_{a b}\right)
$$

Now, one has to shift the contour of integration over the loop spatial momentum into the complex plane. Due to the exponent $\mathrm{e}^{-i p^{1} L}$, the integral over the shifted contour will be negligible and the main contribution will come from crossing a pole of a Green's function in one of the graphs of Figure 2. This is the crucial point for arriving at Lüscher's corrections. Taking the residue effectively puts the line in question on-shell, thus reducing the two-dimensional loop integral to a single dimensional one. It is very convenient to eliminate the spatial momentum using the mass shell condition, and leave the last integral over Euclidean energy which we denote by $q$. The on-shell condition becomes

$$
q^{2}+\varepsilon\left(p^{1}\right)^{2}=0
$$




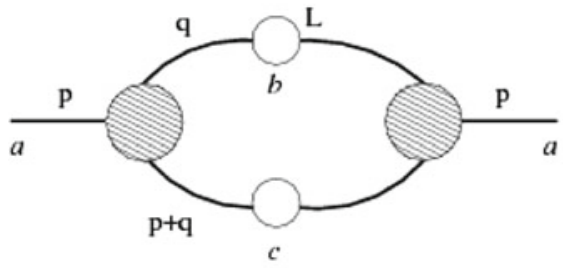

(a)

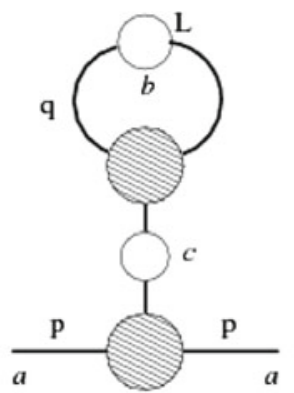

(b)

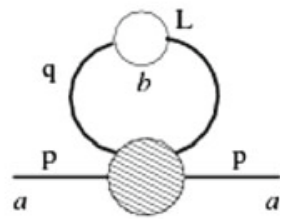

(c)

Figure 2. The graphs giving a leading finite size correction to the self energy: a $I_{a b c}$, b $J_{a b c}$, c $K_{a b}$. The filled circles are the vertex functions $\Gamma$, empty circles represent the 2-point Green's function. The letter $L$ represents the factor of $\mathrm{e}^{-i q^{1} L}$ and the letters in italics label the type of particles.

which in the case of the $\operatorname{Ad} S_{5} \times S^{5}$ superstring theory leads to

$$
p^{1}=-i 2 \operatorname{arcsinh} \frac{\sqrt{1+q^{2}}}{4 g}
$$

Plugging this back into the exponential factor $\mathrm{e}^{-i p^{1} L}$ leads to the term which governs the overall magnitude of the Lüscher correction

$$
\mathrm{e}^{-i p^{1} L}=\mathrm{e}^{-L \cdot E_{\mathrm{TBA}}(q)}=\mathrm{e}^{-L \cdot 2 \operatorname{arcsinh} \frac{\sqrt{1+q^{2}}}{4 g}}
$$

We will analyze the physical meaning of this formula in Section 3.

The mass shell condition has also another, equally important, consequence. Since the line is on-shell, in the integrand we may cut it open thus effectively transforming the graphs of the 2-point 1PI self energy into those of a 4-point forward Green's function. Keeping track of all the necessary factors gives

$$
\Sigma_{L}=\int \frac{\mathrm{d} q}{2 \pi} \frac{i}{\varepsilon^{2}\left(p^{1}\right)^{\prime}} \cdot \mathrm{e}^{-L \cdot E_{\mathrm{TBA}}(q)} \cdot \sum_{b}(-1)^{F_{b}} G_{a b a b}(-p,-q, p, q)
$$

The $p$ appearing in the argument of $G_{a b a b}$ is the spatial momentum of the physical particle, while $q$ is the Euclidean energy of the virtual one. In the final step, one links the 4-point forward Green's function with the forward $S$-matrix element arriving at Lüscher's $F$-term formula generalized to a generic dispersion relation:

$$
\delta \varepsilon_{a}^{F}=-\int_{-\infty}^{\infty} \frac{\mathrm{d} q}{2 \pi}\left(1-\frac{\varepsilon^{\prime}(p)}{\varepsilon^{\prime}(q)}\right) \cdot e^{L \cdot E_{T B A}(q)} \cdot \sum_{b}(-1)^{F_{b}}\left(S_{b a}^{b a}(q, p)-1\right)
$$

with the same conventions for the arguments of $\varepsilon^{\prime}$ and $S_{b a}^{b a}$ as described below (2.11). The $\mu$-term arises in the process of shifting the contours by localizing on a residue of the above formula. It is thus given just by the residue of (2.12). For 
further details consult [13]. Let us mention that for relativistic theories one can perform a more detailed analysis concerning the contribution of $\mu$-terms [11]. In particular, $\mu$-terms are expected to contribute only if, in the spacetime diagram shown in Figure 1, both particles move forward in time (i.e. have positive real part of the energy). This analysis has not been done rigorously for the $A d S_{5} \times S^{5}$ case.

The diagrammatic derivation presented above is very general and does not require integrability. Moreover, the difference between a theory with diagonal and non-diagonal scattering is quite trivial. One can go from the simpler case of a single particle species to the most general case of nondiagonal scattering just by substituting the scalar $S$-matrix by an appropriate supertrace of the nondiagonal $S$ matrix. Generalizing this property to multiparticle states leads to a simple shortcut for obtaining multiparticle Lüscher corrections - one can first obtain the formulas for a simple theory with a single particle in the spectrum, and then generalize to the generic case by trading the product of the scalar $S$-matrices for a supertrace of the product of the nondiagonal ones. We will present this derivation in the following section.

\subsection{MULTIPARTICLE LÜSCHER CORRECTIONS FROM TBA}

In this section, we will show how multiparticle Lüscher corrections arise from the Thermodynamic Bethe Ansatz. Here, we will be able to obtain these more powerful results using significantly stronger assumptions. Particularly, we will assume that the theory in question is integrable with diagonal scattering. Then, as explained above, we will use the expected very universal dependence of Lüscher corrections on the $S$-matrix to conjecture the general versions valid for any integrable theory with a nondiagonal $S$-matrix (for which TBA equations are much more complicated).

As explained in [8], TBA equations are derived by trading the complicated problem of finding the (ground state) energy of the theory at finite volume for the much simpler one of computing a thermal partition function of the theory with space and time interchanged through a double Wick rotation. In the latter case, since one is dealing with the theory at almost infinite volume, Bethe ansatz is exact and can be used to evaluate the partition function. Hence, the energies and momenta in the following are those of the spacetime interchanged one ( $a k a$ mirror theory) related to the energy and momentum of the original theory through

$$
\tilde{E}=i p \quad \tilde{p}=i E
$$

The ground state TBA equation for the theory with a single type of particle takes the form

$$
\varepsilon(z)=L \tilde{E}(z)+\int \frac{\mathrm{d} w}{2 \pi i}\left(\partial_{w} \log S(w, z)\right) \log \left(1+\mathrm{e}^{-\varepsilon(w)}\right)
$$


and the ground state energy is obtained from the solution $\varepsilon(z)$ through

$$
E=-\int \frac{\mathrm{d} z}{2 \pi} \tilde{p}^{\prime}(z) \log \left(1+\mathrm{e}^{-\varepsilon(z)}\right)
$$

In order to describe excited states, one uses an analytical continuation trick due to Dorey and Tateo [14] that essentially introduces additional source terms into (2.14). These source terms are generated by singularities of the integrand $1+$ $\mathrm{e}^{-\varepsilon\left(z_{i}\right)}=0$, which, through integration by parts and an evaluation through residues give rise to additional source terms on the r.h.s. of (2.14)

$$
\begin{aligned}
\varepsilon(z)= & L \tilde{E}+\log S\left(z_{1}, z\right)+\log S\left(z_{2}, z\right) \\
& +\int \frac{\mathrm{d} w}{2 \pi i}\left(\partial_{w} \log S(w, z)\right) \log \left(1+\mathrm{e}^{-\varepsilon(w)}\right)
\end{aligned}
$$

and additional contributions to the energy

$$
E=E\left(z_{1}\right)+E\left(z_{2}\right)-\int \frac{\mathrm{d} z}{2 \pi} \tilde{p}^{\prime}(z) \log \left(1+\mathrm{e}^{-\varepsilon(z)}\right)
$$

where we quote the result with just two additional singularities.

It is quite nontrivial what kind of source terms to introduce for the theory at hand. If a theory does not have bound states and $\mu$-terms, the rule of thumb is that for each physical particle a single source term has to be included (this happens in the case of e.g. the sinh-Gordon model). On the other hand, for a theory with $\mu$-terms, like the SLYM, at least two source terms correspond to a single physical particle (see $[14,15])$.

Now in order to obtain the Lüscher corrections, we have to perform a large volume expansion of these equations. Solving (2.16) by iteration, neglecting the integral term and inserting this approximation into the energy formula gives

$$
\begin{aligned}
E & =E\left(z_{1}\right)+E\left(z_{2}\right)-\int \frac{\mathrm{d} z}{2 \pi} \tilde{p}^{\prime} \mathrm{e}^{-L \tilde{E}(z)} \frac{1}{S\left(z_{1}, z\right) S\left(z_{2}, z\right)} \\
& =E\left(z_{1}\right)+E\left(z_{2}\right)-\int \frac{\mathrm{d} q}{2 \pi} \mathrm{e}^{-L \tilde{E}(q)} S\left(z, z_{1}\right) S\left(z, z_{2}\right)
\end{aligned}
$$

We recognize at once an integral of the $F$-term type (with $q \equiv \tilde{p}$ ) in addition to the sum of energies of the individual particles. There is a subtlety here, namely one has to dynamically impose the equations for the positions of the singularities

$$
1+\mathrm{e}^{-\varepsilon\left(z_{i}\right)}=0
$$

If we insert here the same approximation as we have just used in the formula for the energy, we will recover the Bethe equations

$$
\mathrm{e}^{i L p_{1}}=S\left(p_{1}, p_{2}\right)
$$


However, in Lüscher's corrections we should keep all leading exponential terms. Therefore, for the quantization condition (2.19), we have to use also the first nontrivial iteration of (2.16). This will give rise to modifications of the Bethe quantization conditions. The quantization conditions $\varepsilon\left(z_{i}\right)=i \pi$ becomes

$$
\begin{aligned}
& 0=\underbrace{\log \left\{\mathrm{e}^{i L p_{1}} S\left(z_{2}, z_{1}\right)\right\}}_{B Y_{1}}+\underbrace{\int \frac{\mathrm{d} w}{2 \pi i}\left(\partial_{w} S\left(w, z_{1}\right)\right) S\left(w, z_{2}\right) \mathrm{e}^{-L \tilde{E}(w)}}_{\Phi_{1}} \\
& 0=\underbrace{\log \left\{\mathrm{e}^{i L p_{2}} S\left(z_{1}, z_{2}\right)\right\}}_{B Y_{2}}+\underbrace{\int \frac{\mathrm{d} w}{2 \pi i} S\left(w, z_{1}\right)\left(\partial_{w} S\left(w, z_{2}\right)\right) \mathrm{e}^{-L \tilde{E}(w)}}_{\Phi_{2}}
\end{aligned}
$$

Since the integrals are exponentially small we may solve these equations in terms of corrections to the Asymptotic Bethe Ansatz (ABA) giving

$$
\begin{aligned}
& \frac{\partial B Y_{1}}{\partial p_{1}} \delta p_{1}+\frac{\partial B Y_{1}}{\partial p_{2}} \delta p_{2}+\Phi_{1}=0 \\
& \frac{\partial B Y_{2}}{\partial p_{1}} \delta p_{1}+\frac{\partial B Y_{2}}{\partial p_{2}} \delta p_{2}+\Phi_{2}=0
\end{aligned}
$$

The final formula for the energy thus takes the form

$$
E=E\left(p_{1}\right)+E\left(p_{2}\right)+E^{\prime}\left(p_{1}\right) \delta p_{1}+E^{\prime}\left(p_{2}\right) \delta p_{2}-\int \frac{\mathrm{d} q}{2 \pi} \mathrm{e}^{-L \tilde{E}} S\left(z, z_{1}\right) S\left(z, z_{2}\right)
$$

For nondiagonal scattering, we expect that the above formula will get modified just by exchanging the products of scalar $S$-matrices by a supertrace of a product of real matrix $S$-matrices. This generalization has been proposed in [39]. In the $F$-term integrand we will thus get the transfer matrix (c.f. [9]) or more precisely its eigenvalue $^{1}$

$$
\mathrm{e}^{i \delta\left(\tilde{p} \mid p_{1}, \ldots, p_{N}\right)}=(-1)^{F}\left[S_{a_{1} a}^{a_{2} a}\left(\tilde{p}, p_{1}\right) S_{a_{2} a}^{a_{3} a}\left(\tilde{p}, p_{2}\right) \ldots S_{a_{N} a}^{a_{1} a}\left(\tilde{p}, p_{N}\right)\right]
$$

where we also substituted the complex rapidities used earlier by more physical momenta. The BY condition reads as

$$
2 n_{k} \pi=B Y_{k}\left(p_{1}, \ldots p_{n}\right)+\delta \Phi_{k}=p_{k} L-i \log \left[\prod_{k \neq j} S_{a a}^{a a}\left(p_{k}, p_{j}\right)\right]+\delta \Phi_{k}
$$

with the correction to these equations given by

$$
\delta \Phi_{k}=-\int_{-\infty}^{\infty} \frac{\mathrm{d} \tilde{p}}{2 \pi}(-1)^{F}\left[S_{a_{1} a}^{a_{2} a}\left(\tilde{p}, p_{1}\right) \ldots \frac{\partial S_{a_{k} a}^{a_{k+1} a}\left(\tilde{p}, p_{k}\right)}{\partial \tilde{p}} \ldots S_{a_{N} a}^{a_{1} a}\left(\tilde{p}, p_{N}\right)\right] \mathrm{e}^{-\tilde{\varepsilon}_{a_{1}}(\tilde{p}) L}
$$

\footnotetext{
${ }^{1}$ We present below the case when the physical particles forming the multiparticle state scatter between themselves diagonally.
} 
The final correction then reads as

$$
\begin{aligned}
E(L)= & \sum_{k} \varepsilon\left(p_{k}\right)-\sum_{j, k} \frac{d \varepsilon\left(p_{k}\right)}{d p_{k}}\left(\frac{\delta B Y_{k}}{\delta p_{j}}\right)^{-1} \delta \Phi_{j} \\
& -\int_{-\infty}^{\infty} \frac{\mathrm{d} \tilde{p}}{2 \pi} \sum_{a_{1}, \ldots, a_{N}}(-1)^{F}\left[S_{a_{1} a}^{a_{2} a}\left(\tilde{p}, p_{1}\right) S_{a_{2} a}^{a_{3} a}\left(\tilde{p}, p_{2}\right) \ldots S_{a_{N} a}^{a_{1} a}\left(p, p_{N}\right)\right] \mathrm{e}^{-\varepsilon_{a_{1}}(\tilde{p}) L}
\end{aligned}
$$

For theories with $\mu$-terms, we expect that the corresponding $\mu$-terms will be obtained by localizing the integrals on the poles of the $S$-matrix.

\subsection{POISSON RESUMMATION OF FLUCTUATIONS}

In this section we will present a simple, very physical, derivation of Lüscher's $F$-term formula from a summation over quadratic fluctuations. Although this approach requires the most restrictive assumptions, it is quite intuitive and gives a new perspective on the origin of Lüscher's corrections.

For simplicity, we will just present the derivation for a particle with vanishing momentum, analogous to Lüscher's original formulas. A more general case is treated $^{2}$ in [16].

Consider a soliton at rest which is put on a very large cylinder, so large that we may neglect the effect of the deformation of the solution. Now a small fluctuation very far from the soliton will just be an excitation of the vacuum, so can be treated as another soliton ${ }^{3}$ (more precisely a single particle state). This small 'fluctuation' soliton will scatter on the stationary one and will get a phase shift expressible in terms of the $S$-matrix (which we assume here to be diagonal)

$$
S_{b a}^{b a}(k, p)=\mathrm{e}^{i \delta_{b a}(k, p)}
$$

Due to the finite size of the cylinder, the momentum of the 'fluctuation' soliton will have to be quantized giving

$$
k_{n}=\frac{2 \pi n}{L}+\frac{\delta_{b}\left(k_{n}\right)}{L}
$$

We now have to perform a summation over the energies of the fluctuations

$$
\delta \varepsilon_{\text {naive }}=\frac{1}{2} \sum_{b} \sum_{n=-\infty}^{\infty}(-1)^{F_{b}}\left(\varepsilon\left(k_{n}\right)-\varepsilon\left(k_{n}^{(0)}\right)\right)
$$

where the energies of fluctuations around the vacuum (with $k_{n}^{(0)}=2 \pi n / L$ ) have been subtracted out.

\footnotetext{
${ }^{2}$ In Ref. [16], a minus sign will have to be included in the second term in Equation (13) there.

${ }^{3}$ Here we use 'soliton' as a generic term which includes e.g. 'breathers' in the sine-Gordon model.
} 
The key result of [16] is that Lüscher's corrections are exactly the leading exponential terms $(m= \pm 1)$ in the Poisson resummation

$$
\sum_{n=-\infty}^{\infty} F\left(\frac{2 \pi n}{L}\right)=\frac{L}{2 \pi} \sum_{m=-\infty}^{\infty} \int_{-\infty}^{+\infty} F(t) \mathrm{e}^{-i m L t} \mathrm{~d} t
$$

of (2.32). The relevant terms will be

$$
\delta \varepsilon=\frac{L}{4 \pi} R e \int_{-\infty}^{\infty} \mathrm{e}^{i L t}(\varepsilon(k(t))-\varepsilon(t)) \mathrm{d} t
$$

where $k(t)=t+\delta(k(t)) / L$ is the quantization condition, the solution of which we do not need explicitly. Now, after a sequence of integration by parts and a simple change of variables (see [16] for details) we can rewrite (2.34) as

$$
\begin{aligned}
\delta \varepsilon & =\frac{1}{4 \pi i} \operatorname{Re} \int_{-\infty}^{+\infty} \mathrm{e}^{-i L k}\left(\mathrm{e}^{i \delta(k)}-1\right) \varepsilon^{\prime}(k) \mathrm{d} k \\
& =\frac{1}{4 \pi i} \operatorname{Re} \int_{-\infty}^{+\infty} \mathrm{e}^{-i L k}\left(S_{b a}^{b a}(k, p)-1\right) \varepsilon^{\prime}(k) \mathrm{d} k
\end{aligned}
$$

which is essentially Lüscher's $F$-term but evaluated on the physical line. We should now shift the contour to ensure that the exponent is strictly real and decreasing at infinity giving rise to Lüscher's corrections. Here the boundary terms require a case by case analysis. Also $\mu$-terms may be generated when in the process of shifting the contour we would encounter bound state poles. The above derivation shows that evaluating Lüscher $F$-term contributions is equivalent to computing directly 1-loop energy shifts around the corresponding classical solution. Although one has to be careful in this interpretation when one evaluates the phase shifts (2.30) exactly and not only semiclassically.

\section{Applications of Generalized Lüscher's Corrections in the AdS/CFT Correspondence}

In this section, we will briefly review various applications of generalized Lüscher's corrections in the context of the integrable worldsheet QFT of the superstring in $A d S_{5} \times S^{5}$. Due to the AdS/CFT correspondence, the energy levels of this theory (energies of string states) are identified with the anomalous dimensions of the corresponding gauge theory operators. In this way, the intrinsically two-dimensional methods may be applied to the four-dimensional $\mathcal{N}=4$ SYM theory.

Before we review the obtained results, let us first discuss the generic magnitude of Lüscher corrections in this context. 
As we saw from the derivations, the order of magnitude of the $F$-term formula is essentially governed by the exponential factor [17]

$$
\mathrm{e}^{-L \tilde{E}(\tilde{p})}
$$

where $\tilde{E}$ and $\tilde{p} \equiv q$ are the energy and momentum of a theory with a double Wick rotation exchanging space and time - called 'mirror theory' [18]. For the case at hand we have

$$
\mathrm{e}^{-L \cdot 2 \operatorname{arcsinh}} \frac{\sqrt{Q^{2}+q^{2}}}{4 g}
$$

where $Q=1$ corresponds to the fundamental particle (magnon) and $Q>1$ labels the bound states of the theory.

In the strong coupling limit, this expression becomes

$$
\left.\mathrm{e}^{-Q \frac{L}{2 g}}\right|_{Q=1}=\mathrm{e}^{-\frac{2 \pi L}{\sqrt{\lambda}}}
$$

which is the typical finite size fluctuation effect observed for spinning strings $[19,20]$. We also see that at strong coupling, the contribution of bound states is exponentially suppressed, so one can just consider the fundamental magnons circulating around the cylinder.

The $\mu$-term, which arises from the $F$-term by taking residues also appears at strong coupling. It's magnitude at strong coupling for a single magnon can be estimated to be

$$
e^{-\frac{2 \pi J}{\sqrt{\lambda} \sin \frac{p}{2}}}
$$

where $p$ is the momentum of the physical magnon. We see that the exponential term gives a stronger suppression than the $F$-term, however, the terms differ in the scaling of the prefactor with the coupling. The $F$-term is associated with quantum effects, while the $\mu$-term appears already in the classical contribution hence the $F$-term is suppressed by a factor of $\sqrt{\lambda}$ w.r.t. the $\mu$-term. Let us note that the link between $\mu$-terms and classical solutions is still to a large extent not understood. We may get another qualitative estimate from the formula (3.4) for classical finitegap solutions which may be considered to arise in the worldsheet theory as a state of very many particles, each of which will presumably have a very small momentum. Then (3.4) suggests that the $\mu$-term should be completely negligible for such states.

At weak coupling, we obtain a quite different formula

$$
\frac{\# g^{2 L}}{\left(Q^{2}+q^{2}\right)^{L}}
$$

Firstly, we see that the effect of the virtual corrections only starts at a certain loop order, from the point of view of gauge theory perturbative expansion. Up to 
this order the Bethe equations are in fact exact. Such a behaviour is wholly due to the nonstandard AdS dispersion relation (2.3). The loop order at which these corrections start to contribute is related to the size of the gauge theory operator in question. This is very good, as just at that order we expect a new class of Feynman graphs to appear in the perturbative computation. These are the socalled 'wrapping corrections' and are given by graphs where, in the computation of a two point function relevant for extracting anomalous dimensions, at least one propagator crosses all vertical legs. From the very start [21] (see also [22]), these graphs were expected not to be described by the ABA. Their identification with (possibly multiple) Lüscher corrections was first proposed in [17].

Secondly, we see that at weak coupling, all bound states contribute at the same order. This makes the computation of wrapping effects at weak coupling more complicated, but at the same time more interesting, as they are sensitive to much finer details of the worldsheet QFT than at strong coupling.

The corrections to Lüscher formulas are very difficult to quantify. Even in the relativistic case there are no formulas for the leading corrections. These would be multiple wrapping graphs and hence a 0 th order estimate of their relative magnitude would be another exponential term. At strong coupling we would thus probably see a mixture of the first double wrapping graphs for magnons with ordinary single wrapping graphs for the first $Q=2$ bound states. At weak coupling, the next wrapping correction would generically have a relative magnitude of $g^{2 L}$ although there might also be factors of $g$ coming from the prefactor which we do not control so the loop order for subleading multiple wrapping corrections is not precisely determined.

Let us finish this section with a brief note on the elusive nature of $\mu$-terms. Physical arguments based on the relativistic spacetime picture of the $\mu$-term diagram, amounting to the requirement that the produced virtual particles propagate forward in time suggests that at weak coupling $\mu$-terms should not appear since the bound state is heavier than the fundamental magnon. Explicit computations for the Konishi operator and twist-2 operators (see Section 3.2 below) confirm this intuition. Yet, at strong coupling the $\mu$-term definitely contributes to the giant magnon finite size dispersion relation. It is still not understood how and when does this occur, especially in terms of the proposed exact TBA formulations.

\subsection{STRONG COUPLING RESULTS}

An excitation of the worldsheet theory with momentum $p \sim \mathcal{O}(1)$ has an energy which scales as $\sqrt{\lambda}$ characteristic of a classical string solution. Such a solution has been found in [23] and is called the 'giant magnon'. Subsequently, corrections to its energy were found when the excitation was considered on a cylinder of finite size $J$. The resulting correction was evaluated from the deformed classical solution in $[24,25]$ to be 


$$
\delta E_{\text {string }}=-\frac{\sqrt{\lambda}}{\pi} \cdot \frac{4}{\mathrm{e}^{2}} \cdot \sin ^{3} \frac{p}{2} \cdot \mathrm{e}^{-\frac{2 \pi J}{\sqrt{\lambda} \sin \frac{p}{2}}} \equiv-g \cdot \frac{16}{\mathrm{e}^{2}} \cdot \sin ^{3} \frac{p}{2} \cdot \mathrm{e}^{-\frac{2}{g \sin \frac{p}{2}} J}
$$

In [13], the above expression was recovered from Lüscher's corrections. The exponential term is different from the one appearing in the $F$-term formula, however, it turns out that it is exactly the term appearing in the $\mu$-term, when we find the residue of the $F$-term expression at the bound state pole.

The prefactor comes from evaluating the residue of the (super)trace of the forward $S$-matrix at the bound state pole. A very curious feature of the above expression is the contribution of the dressing factor, which, at strong coupling, has an expansion (see [26])

$$
\sigma^{2}=\exp \left(g \chi_{\mathrm{AFS}}+\chi_{\mathrm{HL}}+\sum_{n=2}^{\infty} \frac{1}{g^{n-1}} \chi_{n}\right)
$$

Naively, one may expect that only the first two terms would give a contribution, however it turns out that due to the special kinematics of the bound state pole, all $\chi_{2 n}$ contribute. The evaluation of this contribution is quite nontrivial with a divergent series appearing, which can be resumed using Borel resummation. The result exactly reproduces (3.6).

Among further developments, finite size contributions to dyonic giant magnons were analyzed [27], quantum fluctuations were linked with the $F$-term [16,28], similar computations were also done for giant magnons and dyonic magnons in the ABJM theory [29-33]. In addition finite size corrections were evaluated for open strings (which corresponds to Lüscher corrections in a boundary integrable field theory [34]) [35,36].

One can also analyze Lüscher corrections for classical spinning strings. There the picture is quite different from the giant magnons. The spinning string solutions arise as a superposition of very many excitations, all with very small momenta. So the $\mu$-term exponential factor will be very much suppressed and the dominant correction will arise from the $F$-term. The $F$-term integrand can be evaluated in terms of the transfer matrix directly in terms of the Bethe root distributions describing the spinning string in question. Alternatively, an analysis of these issues have been done from the algebraic curve perspective in [37].

\subsection{WEAK COUPLING RESULTS}

Lüscher's corrections are particularly interesting when applied in the weak coupling regime corresponding to perturbative gauge theory. There, they provide the only calculational method to compute wrapping corrections apart from a direct perturbative computation which usually is prohibitively complicated (see [10]). Calculations based on generalized Lüscher's corrections are typically much simpler and allow to obtain 4- and 5-loop gauge theory results which cannot be obtained using other means. 
From a more theoretical perspective, the agreement of Lüscher corrections with perturbative gauge theory results is interesting as it gives a nontrivial quantitative test of the AdS/CFT correspondence, as well as of our understanding of the fine details of the worldsheet QFT of the $A d S_{5} \times S^{5}$ superstring. Moreover, it is very interesting to realize that the breakdown of the ABA for anomalous dimensions in the four-dimensional gauge theory occurs exactly in a way characteristic of a two-dimensional quantum field theory (and thus characteristic of string theory).

A natural testing ground for these methods is the Konishi operator $\operatorname{tr} \Phi_{i}^{2}$ (or equivalently $\operatorname{tr} X Z X Z-\operatorname{tr} X^{2} Z^{2}, \operatorname{tr} D Z D Z-\operatorname{tr} Z D^{2} Z$ ), which is the shortest operator not protected by supersymmetry.

From the string perspective, it corresponds to a two particle state in the worldsheet QFT on a cylinder of size $J=2$. Despite the fact that $J$ is so small, we may expect to get an exact answer from Lüscher corrections at least at 4- and 5-loop level due to the estimate (3.5). Since at weak coupling all bound states contribute at the same order, we have to perform a summation over all bound states and their polarization states and use the bound state-fundamental magnon $S$-matrix. There is a further subtlety here, which does not appear in relativistic systems. In the physical theory, the bound states discovered in [38] are in the symmetric representation, while states in the antisymmetric representation are unstable. On the other hand, in the mirror theory, the physical bound states are in the antisymmetric representation [18], and in fact it is these antisymmetric bound states which have to be considered when computing Lüscher's corrections.

Performing the computation yields the result for the 4-loop wrapping correction to the anomalous dimension of the Konishi operator [39]:

$$
\Delta_{w}^{(8)}=324+864 \zeta(3)-1440 \zeta(5)
$$

which is in exact agreement with direct perturbative computations using both supergraph techniques [40,41] and component Feynman graphs [42]. The string computation is much simpler as it involves evaluating just the single graph shown in Figure 3.

In another development, wrapping corrections for twist two operators

$\operatorname{tr} Z D^{M} Z+$ permutations

were computed. Here, the main motivation for performing this computation was the fact there are stringent analytical constraints on the structure of the anomalous dimensions $\Delta(M)$ as a function of $M$. In fact the disagreement, at 4 loops, between the behaviour of the Bethe Ansatz $\Delta(M)$ for $M=-1+\omega$ and gauge theory constraints from the BFKL (Balitsky-Fadin-Kuraev-Lipatov) and NLO BFKL equations describing high energy scattering in the Regge limit [43-45] were the first quantitative indication that the ABA breaks down [46].

In [47], the anomalous dimensions of twist two operators were evaluated at 4-loop level using Lüscher corrections for an $M$-particle state. The wrapping 


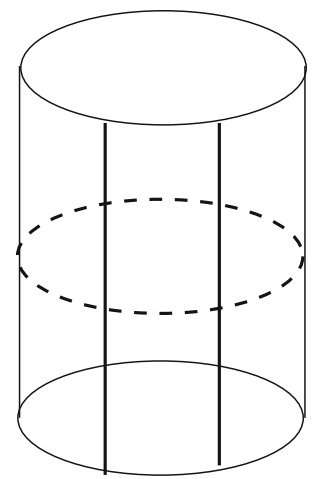

Figure 3. The single Lüscher graph entering the computation of the four loop Konishi anomalous dimension.

correction was found to exactly compensate the mismatch between the Bethe Ansatz and BFKL expectations.

Subsequently, the leading wrapping corrections for the lowest lying twist-three operators were also determined from Lüscher corrections [48]. These occur at 5-loop level. Another class of operators which was considered were single particle states [49] and the Konishi operator [50] in the $\beta$ deformed theory. These results agree with direct field theoretical computations when available [51,52].

In all the above computations of the leading wrapping corrections, there were significant simplifications. Firstly, the wrapping modifications of the Bethe Ansatz quantization condition did not appear. Secondly, the dressing factor of the $S$-matrix also did not contribute.

Once one moves to subleading perturbative wrapping order (5-loop for Konishi and twist two, and 6-loop for twist three), both of these effects start to play a role. The modification of the Bethe Ansatz quantization is particularly interesting, as it is only in its derivation that the convolution terms in TBA equations contribute. In contrast to the simple single component TBA equation presented here, the structure of the TBA equations proposed for the $A d S_{5} \times S^{5}$ system is very complicated [53-57]. So Lüscher corrections may be a nontrivial cross-check for these proposals. In addition, due to the kinematics of the scattering between the physical particle and the mirror particle, it turns out that already at 5 loops, an infinite set of coefficients of the BES/BHL dressing phase contributes to the answer.

A key difficulty in performing such a computation is the possibility of testing the answer. Fortunately, we have at our disposal two independent consistency checks. Firstly, at weak coupling we do not expect the appearance of $\mu$-terms which implies that a sum over residues of certain dynamical poles in the integrand has to cancel after summing over all bound states. Secondly, the transcendental structure of the final answer should be quite simple, while the subexpressions involve very complicated expressions which should cancel out in the final answer. In addition, for the case of twist two operators, one can use the numerous 
stringent constraints on the analytic structure coming from BFKL, NLO BFKL, reciprocity etc.

In [58], the 5-loop wrapping correction to the Konishi anomalous dimension was derived

$$
\Delta_{w}^{(10)}=-11340+2592 \zeta(3)-5184 \zeta(3)^{2}-11520 \zeta(5)+30240 \zeta(7)
$$

while in [59] a tour-de-force computation was performed for twist two operators at 5-loops. Subsequently twist three operators were also considered at subleading wrapping order in [60].

Recently, the 5-loop result coming from Lüscher corrections was confirmed by expanding the exact TBA equations at large volume first numerically [61], and then analytically [62,63]. Finally, subsubleading (6-loop) wrapping corrections were considered for single impurity operators in the $\beta$ deformed theory [64].

\section{Summary and Outlook}

Lüscher's corrections situate themselves in the middle ground between Bethe Ansatz and a full fledged solution of two-dimensional integrable quantum field theories in the guise of Thermodynamic Bethe Ansatz or nonlinear integral equations. They encode effects of an explicitly quantum field theoretical nature, namely virtual corrections associated with the topology of a cylinder. In this way, Lüscher's corrections may be seen to differentiate between spin chain like systems, where the Bethe Ansatz is exact and quantum field theories, for which the Bethe Ansatz is only a large volume approximation.

In this review, we have presented various ways of arriving at Lüscher's corrections, some of them more or less rigorous, others more conjectural. The fact that the methods are quite different one from the other serves as an important cross check of these results. It would be, however, quite interesting to extend some of these methods in various directions e.g. the diagrammatic calculations to multiparticle states and subleading wrapping. Recently, the multiparticle Lüscher corrections proposed in [39] were tested in [61-63,65,66]. It would be interesting to obtain some kind of universal understanding how the structure necessary for Lüscher corrections is encoded in the very complicated nondiagonal TBA systems.

With respect to the concrete applications of Lüscher corrections in the AdS/CFT correspondence there are still some loose ends like the rather mysterious formula for the finite size corrections of the giant magnon in the $\beta$ deformed theory [67]. Apart from that, the agreement between the computations based on Lüscher corrections, which typically involve a single graph, and the very complicated 4-loop gauge theory computations involving hundreds or even many thousands of graphs suggests that there is some very nontrivial hidden structure in the perturbative expansion. It would be very interesting to understand whether it could be understood in any explicit way. 


\section{Acknowledgements}

This work was supported by Polish science funds as a research project N N202 105136 (2009-2011).

Open Access This article is distributed under the terms of the Creative Commons Attribution Noncommercial License which permits any noncommercial use, distribution, and reproduction in any medium, provided the original author(s) and source are credited.

\section{References}

1. Staudacher, M.: Review of AdS/CFT integrability, Chapter III.1: Bethe Ansätze and the $R$-matrix formalism. Lett. Math. Phys. Published in this volume. arxiv:1012.3990

2. Ahn, C., Nepomechie, R.: Review of AdS/CFT integrability, Chapter III.2: exact world-sheet $S$-matrix. Lett. Math. Phys. Published in this volume. arxiv:1012.3991

3. Luscher, M.: Volume dependence of the energy spectrum in massive quantum field theories. 1. Stable particle states. Commun. Math. Phys. 104, 177 (1986)

4. Rej, A., Serban, D., Staudacher, M.: Planar $\mathcal{N}=4$ gauge theory and the Hubbard model. JHEP 0603, 018 (2006). hep-th/0512077

5. Zamolodchikov, A.B.: Thermodynamic Bethe Ansatz in relativistic models. Scaling three state potts and Lee-Yang models. Nucl. Phys. B 342, 695 (1990)

6. Destri, C., De Vega, H.J.: Unified approach to thermodynamic Bethe Ansatz and finite size corrections for lattice models and field theories. Nucl. Phys. B 438, 413 (1995). hep-th/9407117

7. Fioravanti, D., Mariottini, A., Quattrini, E., Ravanini, F.: Excited state Destri-De Vega equation for sine-Gordon and restricted sine-Gordon models. Phys. Lett. B 390, 243 (1997). hep-th/9608091

8. Bajnok, Z.: Review of AdS/CFT integrability, Chapter III.6: thermodynamic Bethe Ansatz. Lett. Math. Phys. Published in this volume. arxiv:1012.3995

9. Kazakov, V., Gromov, N.: Review of AdS/CFT Integrability, Chapter III.7: Hirota dynamics for quantum integrability. Lett. Math. Phys. Published in this volume. arxiv: 1012.3996

10. Sieg, C.: Review of AdS/CFT integrability, chapter I.2: the spectrum from perturbative gauge theory. Lett. Math. Phys. Published in this volume. arxiv:1012.3984

11. Klassen, T.R., Melzer, E.: On the relation between scattering amplitudes and finite size mass corrections in QFT. Nucl. Phys. B 362, 329 (1991)

12. Arutyunov, G., Frolov, S.: Foundations of the $\operatorname{AdS}_{5} \times \mathrm{S}^{5}$ superstring. Part I. J. Phys. A 42, 254003 (2009), arxiv:0901.4937

13. Janik, R.A., Lukowski, T.: Wrapping interactions at strong coupling - the giant magnon. Phys. Rev. D 76, 126008 (2007). arxiv:0708.2208

14. Dorey, P., Tateo, R.: Excited states by analytic continuation of TBA equations. Nucl. Phys. B 482, 639 (1996). hep-th/9607167

15. Dorey, P., Tateo, R.: Excited states in some simple perturbed conformal field theories. Nucl. Phys. B 515, 575 (1998). hep-th/9706140

16. Heller, M.P., Janik, R.A., Lukowski, T.: A new derivation of Lüscher $F$-term and fluctuations around the giant magnon. JHEP 0806, 036 (2008). arxiv:0801.4463

17. Ambjorn, J., Janik, R.A., Kristjansen, C.: Wrapping interactions and a new source of corrections to the spin-chain/string duality. Nucl. Phys. B 736, 288 (2006). hepth/0510171

18. Arutyunov, G., Frolov, S.: On string $S$-matrix, bound states and TBA. JHEP 0712, 024 (2007). arxiv:0710.1568 
19. Schafer-Nameki, S.: Exact expressions for quantum corrections to spinning strings. Phys. Lett. B 639, 571 (2006). hep-th/0602214

20. Schafer-Nameki, S., Zamaklar, M., Zarembo, K.: How accurate is the quantum string Bethe ansatz? JHEP 0612, 020 (2006). hep-th/0610250

21. Beisert, N., Dippel, V., Staudacher, M.: A novel long range spin chain and planar $\mathcal{N}=4$ super Yang-Mills. JHEP 0407, 075 (2004). hep-th/0405001

22. Sieg, C., Torrielli, A.: Wrapping interactions and the genus expansion of the 2-point function of composite operators. Nucl. Phys. B 723, 3 (2005). hep-th/0505071

23. Hofman, D.M., Maldacena, J.M.: Giant magnons. J. Phys. A 39, 13095 (2006). hepth/0604135

24. Arutyunov, G., Frolov, S., Zamaklar, M.: Finite-size effects from giant magnons. Nucl. Phys. B 778, 1 (2007). hep-th/0606126

25. Astolfi, D., Forini, V.,. Grignani, G., Semenoff, G.W.: Gauge invariant finite size spectrum of the giant magnon. Phys. Lett. B 651, 329 (2007). hep-th/0702043

26. Vieira, P., Volin, D.: Review of AdS/CFT integrability, Chapter III.3: the dressing factor. Lett. Math. Phys. Published in this volume. arxiv:1012.3992

27. Hatsuda, Y., Suzuki, R.: Finite-size effects for dyonic giant magnons. Nucl. Phys. B 800, 349 (2008). arxiv:0801.0747

28. Gromov, N., Schafer-Nameki, S., Vieira, P.: Quantum wrapped giant magnon. Phys. Rev. D 78, 026006 (2008). arxiv:0801.3671

29. Grignani, G., Harmark, T., Orselli, M., Semenoff, G.W.: Finite size giant magnons in the string dual of $N=6$ superconformal Chern-Simons theory. JHEP 0812, 008 (2008). arXiv:0807.0205

30. Bombardelli, D., Fioravanti, D.: Finite-size corrections of the $\mathrm{CP}^{3}$ giant magnons: the Lüscher terms. JHEP 0907, 034 (2009). arxiv:0810.0704

31. Lukowski, T., Sax, O.O.: Finite size giant magnons in the $\mathrm{SU}(2) \times \mathrm{SU}(2)$ sector of $\mathrm{AdS}_{4} \times \mathrm{CP}^{3}$. JHEP 0812, 073 (2008). arxiv:0810.1246

32. Ahn, C., Bozhilov, P.: Finite-size effect of the dyonic giant magnons in $\mathcal{N}=6$ super Chern-Simons theory. Phys. Rev. D 79, 046008 (2009). arxiv:0810.2079

33. Ahn, C., Kim, M., Lee, B.H.: Quantum finite-size effects for dyonic magnons in the $\mathrm{AdS}_{4} \times \mathrm{CP}^{3}$. JHEP 1009, 062 (2010). arxiv:1007.1598

34. Bajnok, Z., Palla, L., Takacs, G.: Finite size effects in quantum field theories with boundary from scattering data. Nucl. Phys. B 716, 519 (2005). hep-th/0412192

35. Correa, D.H., Young, C.A.S.: Finite size corrections for open strings/open chains in planar AdS/CFT. JHEP 0908, 097 (2009). arxiv:0905.1700

36. Bajnok, Z., Palla, L.: Boundary finite size corrections for multiparticle states and planar AdS/CFT. arxiv: 1010.5617

37. Gromov, N., Schafer-Nameki, S., Vieira, P.: Efficient precision quantization in AdS/CFT. JHEP 0812, 013 (2008). arxiv:0807.4752

38. Dorey, N.: Magnon bound states and the AdS/CFT correspondence. J. Phys. A 39, 13119 (2006). hep-th/0604175

39. Bajnok, Z., Janik, R.A.: Four-loop perturbative Konishi from strings and finite size effects for multiparticle states. Nucl. Phys. B 807, 625 (2009). arxiv:0807.0399

40. Fiamberti, F., Santambrogio, A., Sieg, C., Zanon, D.: Wrapping at four loops in $\mathcal{N}=4$ SYM. Phys. Lett. B 666, 100 (2008). arxiv:0712.3522

41. Fiamberti, F., Santambrogio, A., Sieg, C., Zanon, D.: Anomalous dimension with wrapping at four loops in $\mathcal{N}=4$ SYM. Nucl. Phys. B 805, 231 (2008). arxiv:0806.2095

42. Velizhanin, V.N.: The four-loop anomalous dimension of the Konishi operator in $\mathrm{N}=4$ supersymmetric Yang-Mills theory. JETP Lett. 89, 6 (2009). arxiv:0808.3832 
43. Lipatov, L.N.: Reggeization of the vector meson and the vacuum singularity in nonabelian gauge theories. Sov. J. Nucl. Phys. 23, 338 (1976) [Yad. Fiz. 23, 642 (1976)]

44. Kuraev, E.A., Lipatov, L.N., Fadin, V.S.: The Pomeranchuk singularity in nonabelian gauge theories. Sov. Phys. JETP 45, 199 (1977) [Zh. Eksp. Teor. Fiz. 72, 377 (1977)]

45. Balitsky, I.I., Lipatov, L.N.: The Pomeranchuk singularity in quantum chromodynamics. Sov. J. Nucl. Phys. 28, 822 (1978) [Yad. Fiz. 28, 1597 (1978)]

46. Kotikov, A.V., Lipatov, L.N., Rej, A., Staudacher, M., Velizhanin, V.N.: Dressing and wrapping. J. Stat. Mech. 0710, P10003 (2007). arxiv:0704.3586

47. Bajnok, Z., Janik, R.A., Lukowski, T.: Four loop twist two, BFKL, wrapping and strings. Nucl. Phys. B 816, 376 (2009). arxiv:0811.4448

48. Beccaria, M., Forini, V., Lukowski, T., Zieme, S.: Twist-three at five loops, Bethe Ansatz and wrapping. JHEP 0903, 129 (2009). arxiv:0901.4864

49. Beccaria, M., De Angelis, G.F.: On the wrapping correction to single magnon energy in twisted $N=4$ SYM. Int. J. Mod. Phys. A 24, 5803 (2009). arxiv:0903.0778

50. Ahn, C., Bajnok, Z., Bombardelli, D., Nepomechie, R.I.: Finite-size effect for fourloop Konishi of the beta-deformed $N=4$ SYM. Phys. Lett. B 693, 380 (2010). arxiv:1006.2209

51. Fiamberti, F., Santambrogio, A., Sieg, C., Zanon, D.: Single impurity operators at critical wrapping order in the beta-deformed $\mathcal{N}=4$ SYM. JHEP 0908, 034 (2009). arxiv:0811.4594

52. Fiamberti, F., Santambrogio, A., Sieg, C.: Five-loop anomalous dimension at critical wrapping order in $\mathrm{N}=4$ SYM. JHEP 1003, 103 (2010). arxiv:0908.0234

53. Bombardelli, D., Fioravanti, D., Tateo, R.: Thermodynamic Bethe Ansatz for planar AdS/CFT: a proposal. J. Phys. A 42, 375401 (2009). arxiv:0902.3930

54. Gromov, N., Kazakov, V., Vieira, P.: Exact spectrum of anomalous dimensions of planar $N=4$ supersymmetric Yang-Mills theory. Phys. Rev. Lett. 103, 131601 (2009). arxiv:0901.3753

55. Gromov, N., Kazakov, V., Kozak, A., Vieira, P.: Exact spectrum of anomalous dimensions of planar $\mathrm{N}=4$ supersymmetric Yang-Mills theory: TBA and excited states. Lett. Math. Phys. 91, 265 (2010). arxiv:0902.4458

56. Arutyunov, G., Frolov, S.: Thermodynamic Bethe Ansatz for the $\operatorname{AdS}_{5} \times \mathrm{S}^{5}$ mirror model. JHEP 0905, 068 (2009). arxiv:0903.0141

57. Arutyunov, G., Frolov, S.: Simplified TBA equations of the $A d S_{5} \times S^{5}$ mirror model. JHEP 0911, 019 (2009). arxiv:0907.2647

58. Bajnok, Z., Hegedus, A., Janik, R.A., Lukowski, T.: Five loop Konishi from AdS/CFT. Nucl. Phys. B 827, 426 (2010). arxiv:0906.4062

59. Lukowski, T., Rej, A., Velizhanin, V.N.: Five-loop anomalous dimension of twist-two operators. Nucl. Phys. B 831, 105 (2010). arxiv:0912.1624

60. Velizhanin, V.N.: Six-loop anomalous dimension of twist-three operators in $N=4$ SYM. JHEP 1011, 129 (2010). arxiv:1003.4717

61. Arutyunov, G., Frolov, S., Suzuki, R.: Five-loop Konishi from the mirror TBA. JHEP 1004, 069 (2010). arxiv:1002.1711

62. Balog, J., Hegedus, A.: 5-loop Konishi from linearized TBA and the XXX magnet. JHEP 1006, 080 (2010). arxiv:1002.4142

63. Balog, J., Hegedus, A.: The Bajnok-Janik formula and wrapping corrections. JHEP 1009, 107 (2010). arxiv:1003.4303

64. Bajnok, Z., Deeb, O.e.: 6-loop anomalous dimension of a single impurity operator from AdS/CFT and multiple zeta values. JHEP 1101, 054 (2011). arxiv:1010.5606

65. Gromov, N., Kazakov, V., Vieira, P.: Finite volume spectrum of 2D field theories from Hirota dynamics. JHEP 0912, 060 (2009). arxiv:0812.5091 
66. Balog, J., Hegedus, A.: The finite size spectrum of the 2-dimensional $\mathrm{O}(3)$ nonlinear sigma-model. Nucl. Phys. B 829, 425-446 (2010). arxiv:0907.1759

67. Bykov, D.V., Frolov, S.: Giant magnons in TsT-transformed $\mathrm{AdS}_{5} \times \mathrm{S}^{5}$. JHEP 0807, 071 (2008). arxiv:0805.1070 